\title{
Ville-usine, ville industrielle, ville d'entreprise... Introduction à des approches croisées du fait industrialo-urbain
}

Factory town, industrial city, company town... Introduction to crossover

approaches of industrial urban forms

Fabrikstadt, Industriestadt, Firmenstadt ... Einführung in Cross-Ansätze zur

industriell-urbanen Entwicklung

Simon Edelblutte

\section{(2) OpenEdition}

Journals

Édition électronique

URL : http://journals.openedition.org/rge/9332

ISSN : 2108-6478

Éditeur

Association des géographes de l'Est

Référence électronique

Simon Edelblutte, «Ville-usine, ville industrielle, ville d'entreprise...Introduction à des approches croisées du fait industrialo-urbain », Revue Géographique de l'Est [En ligne], vol. 58/3-4 | 2018, mis en ligne le 23 juillet 2020, consulté le 08 septembre 2020. URL : http://journals.openedition.org/rge/9332

Ce document a été généré automatiquement le 8 septembre 2020

Tous droits réservés 


\title{
Ville-usine, ville industrielle, ville d'entreprise...Introduction à des approches croisées du fait industrialo-urbain
}

\author{
Factory town, industrial city, company town... Introduction to crossover \\ approaches of industrial urban forms \\ Fabrikstadt, Industriestadt, Firmenstadt ... Einführung in Cross-Ansätze zur \\ industriell-urbanen Entwicklung
}

Simon Edelblutte

1 Portée par les enjeux économiques, sociaux, environnementaux, politiques et les crises sanitaires, la thématique de la réindustrialisation de la France et, au-delà, du monde développé est, depuis les années 2010, devenue majeure et fait l'objet de nombreuses rencontres ${ }^{1}$, publications et articles, notamment en économie (Levet - dir., 2012 ; Savall et al. - dir., 2013). Miroir de la désindustrialisation beaucoup étudiée et débattue, mais, ainsi que le démontre F. Bost en 2015, avec retard par rapport à la réalité du phénomène, ce retour politique, scientifique, littéraire, médiatique, etc. de l'industrie est inattendu au regard du désintérêt qu'elle subissait dans tous ces domaines dans le dernier quart du XX ${ }^{\text {ème }}$ siècle.

2 En effet, au sortir de Trente Glorieuses, le basculement de la majorité ${ }^{2}$ des économies développées, auparavant dominées par l'industrie, vers une économie dominée par la finance, le commerce et les services a semblé condamner les recherches sur l'industrie, considérée comme quelque peu désuète, représentant un monde ancien, vue comme source de nuisances environnementales et d'aliénation sociale ou encore laissée aux pays émergents. Les écrits, nombreux en sociologie autour de l'idée d'un monde postindustriel (à partir d'A. Touraine en 1969), dominent alors la littérature. Si l'on s'intéresse, au moment fort de la crise industrielle (années 1980 essentiellement) aux thématiques de la reconversion des branches et territoires touchées par ce déclin 
industriel, cet intérêt cède bien vite la place aux thématiques liées à la mondialisation et à ses excès. Ainsi apparaît la nécessité d'inscrire cette mondialisation dans un développement durable, dans un monde où les rassurants secteurs économiques de Fischer et Clarke se brouillent et deviennent inopérants à tel point qu'on se demande, avec S. Montagné-Vilette en 2001, si «le secondaire est [-il] soluble dans le tertiaire?». Dans ce mouvement, l'industrie n'apparaît plus que comme une activité économique un peu en retrait derrière la finance, le commerce, les mobilités, les loisirs... sorte de bruit de fond laissé à des économies émergentes moins regardantes sur la pollution et les conditions de travail.

Cet article introductif revient dans un premier temps sur un préalable essentiel à l'étude des formes industrialo-urbaines: la façon dont les géographes se sont positionnés dans ces évolutions industrialo-économiques et la multiplicité des approches du fait industrialo-urbain. Il insiste ensuite et surtout, en lien avec le thème de ce numéro spécial de la "Revue Géographique de l'Est » (RGE), sur les liens entre l'industrie et la ville. Villes modifiées par l'installation d'usines et des systèmes productifs liés; villes créées de toutes pièces et rapidement par un industriel ou, plus souvent encore, par plusieurs industriels de façon très progressive ; villages ouvriers, voire isolats industriels perdus en milieu rural; villes industrielles, villes usines, villes de compagnie ou d'entreprise... toutes ces notions, toutes ces expressions, gravitent, sans être toujours définies précisément (si tant est que cela soit possible - cf. la conclusion de cet article) autour des liens entre fait urbain, urbanisation, et industrie.

Les huit articles rassemblés dans ce numéro ne clarifieront ni complètement, ni définitivement ces notions, mais y contribueront par la diversité des approches utilisées et du regard de leurs auteures et auteurs.

\section{I - Flux et reflux d'une " géographie industrielle » toujours à questionner}

Dans les mouvements de fond évoqués en introduction, la géographie ne fait pas exception. Si, en pleine révolution industrielle, l'industrie n'a pas été au cœur des préoccupations des géographes vidaliens (Deshaies, 2005), elle est au contraire très étudiée durant les Trente Glorieuses. En effet, dans un contexte de fort développement économique et industriel et de division du monde en grands blocs politiques aux intérêts opposés, la recherche géographique devient beaucoup plus sensible aux problématiques économiques et plus particulièrement industrielles, tant cette dernière activité, associée à l'essor des mines, semble être la racine de tout développement économique et de puissance. La constatation de l'importance de l'industrie dans l'économie, dans la croissance urbaine ou encore dans la hiérarchie des États de la

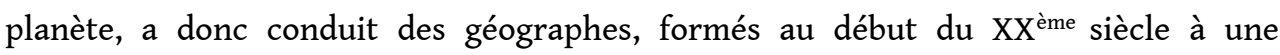
géographie essentiellement régionale et quelque peu figée, à quitter ce cadre et à s'intéresser à la branche industrielle et à sa géographie. L'étude de l'industrie est donc centrale dans les problématiques de géographie économique, la seconde étant quelque peu inféodée à la première, au moins jusqu'aux années 1950-60.

6 De plus, une forte part de la géographie économique de l'époque s'inscrit dans une tendance marxiste productiviste très présente. Ainsi P. George par ailleurs membre du Parti Communiste Français jusqu'en 1960, insiste-t-il en 1978 sur «l'importance du 
développement industriel pour définir géographiquement un espace donné (État ou région)" (p. 92) dans une vision centrée sur "l'homme producteur plus que l'homme habitant" (Gugliemo, 2008, p. 255). Pour G. Benko cette géographie industrielle et économique "s'apparente à un catalogue de la répartition des différentes activités économiques» (2008, p. 32). Il fait ici référence au Précis de géographie économique, de P. George, publié en 1956 et réédité 4 fois jusqu'en 1970 et il précise d'ailleurs que la plupart des manuels de cette époque, comme celui de J.Chardonnet en 1965, par exemple, traitent les activités économiques de cette même façon. Parallèlement, ces localisations industrielles font l'objet de nombreuses théories, issues d'économistes de la fin du XIX ${ }^{\text {ème }}$ au début du XX ème siècle (Launhardt, A. Weber, Palander, etc.). Elles ont pour objet, soit la minimisation des coûts de transport (A. Weber), soit la maximisation du profit (Lösch). Ces théories sont largement reprises par les géographes, comme Cl. Manzagol en 1980, qui construit son ouvrage sur un "examen critique des théories classiques qui précède une analyse des nouvelles approches et la présentation du système industriel » (p. 11).

7 La fin de la guerre froide et l'effondrement de l'Union des Républiques socialistes Soviétiques (URSS), couplée à la crise et la mutation du fordisme (toyotisme, néomanagement, etc.) dans le dernier quart du $\mathrm{XX}^{\text {ème }}$ siècle, signent la quasi disparition de la géographie industrielle telle que pratiquée auparavant. Se développent alors de nouvelles préoccupations dans la discipline, marquée par un tournant social et se préoccupant aussi de l'entreprise et de ses réseaux. Ces évolutions sont amorcées à l'étranger dès les années 1960, comme l'observe en 2003 H.A. Stafford : «dans les années 1960, il y a un mouvement concerté pour l'étude de la façon dont les entreprises organisent l'espace. Cela crée une géographie de l'entreprise » ${ }^{3}$ (p. 5). Ainsi, au Comité Français National de Géographie (CNFG) par exemple, la commission « géographie industrielle » est-elle remplacée, en 1998, par une commission "industrie et emplois», alors que les géographes, de plus en plus, prennent leurs distances avec les théories économicistes dominantes des décennies précédentes. En effet, H.A. Stafford montre qu'il y a

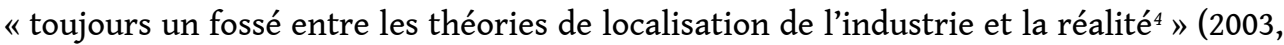
p. 6), tandis que B. Mérenne-Schoumaker (2011) explique que ces théories sont certes des apports fondamentaux au niveau économique, car elles ont montré l'importance de la maîtrise des coûts, mais sont trop simplificatrices au niveau spatial car elles ne prennent pas en compte la rugosité physique et politique de l'espace. L'intérêt de ces théories est ainsi essentiellement de permettre la réflexion quant aux territoires industriels, notamment à l'échelle régionale, mais la complexité de la réalité les rend finalement peu opératoires, notamment aux échelles locales et particulièrement à celle de la ville. Aujourd'hui, écrit d'ailleurs S. Daviet, « les savants calculs de distance et de coût, les belles équations mathématiques, les poids de Launhardt, les triangles d'Alfred Weber ne sont plus présentés comme les clés de lecture de la nouvelle économie » (2005, p. 99).

8 Il semble donc, qu'à la charnière des $\mathrm{XX}^{\text {ème }}$ et XXI ${ }^{\text {ème }}$ siècles, l'étude de l'industrie se dilue de plus en plus dans des approches plus larges ${ }^{5}$, intégrant, aux côtés des préoccupations économiques autrefois clairement dominantes et renouvelées, des préoccupations sociales, culturelles, historiques, territoriales... (Talandier \& Pecqueur, 2018; Boulay \& Grandclément, 2019). Cette dilution autorise aussi, à propos de l'industrie, un élargissement des centres d'intérêt, un renouvellement des thématiques, 
une diversification des sensibilités. Deux exemples, autour des idées de reconversion et de culture industrielles, suivent :

- la crise du fordisme à la fin des Trente Glorieuses a poussé au développement de nombreuses recherches, à partir des années 1980, sur les thématiques des friches industrielles, d'abord vues comme transitoires (Dézert, 1992), puis comme des opportunités (Lamard et al - coll., 2006 ; Janin \& Andres, 2008) et de la reconversion industrielle. Cette dernière, d'abord étudiée à l'échelle seule des sites industriels (Blanc, 1991 ; Grusti, 1991 ; Fagnoni, 2001), est, à l'entrée du XXI İme siècle, étendue à un redéveloppement territorial plus vaste, incluant l'ensemble des territoires construits directement ou indirectement par les industriels (Donze, 2001 ; Daviet, 2006). Il s'agit d'une évolution à la fois multiscalaire - les anciens sites industriels sont reconvertis en fonction des besoins des territoires - et multidimensionnelle - on appuie les projets de reconversion sur les forces des territoires. Ces thématiques ont progressivement été intégrées aux études plus larges sur le renouvellement urbain (Edelblutte, 2006; Veschambre, 2008), intégrant des actions patrimoniales et culturelles dont l'étude est largement développée en histoire (Woronoff, 1989 ; Bergeron \& Dorel-Ferré, 1996 ; Gasnier \& Lamard - dir., 2007) et, plus récemment, en géographie (Fagnoni, 2005 ; Veschambre, 2008; Gravari-Barbas - dir., 2005). Les développements autour de la thématique des villes rétrecissantes (Fol \& Cunnigham-Sabot, 2010 ; Roth, 2011; Grésillon, 2011) concernent aussi beaucoup les villes fortement liées à l'industrie. Il s'agit dans cette évolution d'une vraie reconnaissance et prise en compte du lien entre l'industrie et la ville, pas toujours bien perçu ni étudié et analysé auparavant. Ces thématiques liées au déclin de l'industrie participent donc à une vision large du fait industriel, pas seulement centré sur la production et sa localisation comme lors des Trente Glorieuses. Ainsi, l'industrie est certes une activité économique productive construisant réseaux et territoires actifs, mais a aussi marqué la planète de territoires et de paysages hérités, ayant imprégné les habitants et ainsi construit une culture industrielle ;

- En 2008, G. Benko évoque, à propos de l'évolution récente de la géographie économique, « un tournant culturel » par lequel elle doit se montrer « beaucoup plus attentive aux questions de culture qu'elle ne l'a été par le passé ", car "l'ordre économique du capitalisme contemporain plonge en fait ses racines dans la culture humaine (plutôt que l'inverse)» (p. 42). Cette attention à la culture, déjà bien développé en géographie en général dès le dernier quart du XXème siècle autour de Paul Claval (1995) et de la fondation de la revue " Géographie et Cultures » en 1992, pousse à réinterpréter l'évolution de l'industrie et de ses territoires en y intégrant des approches nouvelles, par les paysages par exemple (Edelblutte, 2010). Cette approche culturelle est particulièrement utile en temps de crise, de reconversion et de gestion des héritages, notamment dans les villes nées de l'industrie. En effet, après une phase de deuil (Grossetti et al, 1998 ; Edelblutte, 2014) pendant laquelle l'identité industrielle de la ville déchue est cachée voire niée, le renouveau passe par une réappropriation de cette identité industrielle qui a sous-tendu l'existence de la ville durant des décennies. Enfin, cette approche permet à la géographie industrielle de dépasser les limites du tout économique, car « il est clair que l'approche culturelle en géographie est une force puissante pour fédérer la discipline tout en améliorant la portée et la polyvalence de la géographie économique » (Stafford, 2003, p. 12).Cette évolution de l'étude du fait industriel, d'une approche majoritairement quantitative, économico-centrée et à l'échelle nationale vers des approches multiples, multiscalaires et transdisciplinaires, trouve un écho dans la variété des contributions abordant la question des liens entre industrie et ville dans ce numéro de la RGE. En effet, en fonction de la spécialité et des pratiques scientifiques de chacun des auteurs, géographes ou non, les approches de l'étude du fait industriel et de ses 
liens avec la ville varient. Ainsi, dans certain cas, la société ouvrière prend plus d'importance que le bâtiment industriel; dans d'autres, l'histoire des luttes sociales moins que celle des théories et idéologies sous-tendant le paternalisme; les déterminants économiques plus que les paysages construits par l'industrie ; les enjeux environnementaux plus que la morphologie urbaine, etc.

Ces approches multiples expliquent aussi, en partie et dans certains cas, les difficultés à bien cerner et identifier certaines formes (s'agit-il plutôt d'une ville-usine planifiée ? d'un village ouvrier? d'une ville industrielle? etc.), voire les contradictions entre les résultats d'une approche et ceux d'une autre. Il est donc essentiel de croiser ces approches pour réussir à identifier les formes urbaines produites ou modifiées par l'industrie. Ceci permet de mieux les prendre en compte dans les problématiques territoriales, aux prismes des enjeux actuels autour de questions environnementales dans le cadre de la transition (Ch. Beaurain \& Ch. Chembessi), de questions patrimoniales liées à l'identité et à la culture (A. Caignet, G. Dorel-Ferré, M. Picon, G. Paşcu, L. Gauduchon), ou encore de questions de gouvernance locale (É. Marochini, A. Brichler).

\section{II - Industrie et formes urbaines : repères et jalons}

Que cela soit dans un contexte de mondialisation débridée ou dans un contexte espéré de relocalisation / réindustrialisation, les usines actuelles, détachées du poids du transport des matières premières et de l'énergie qui contraignait nombre d'entre elles à s'installer près des gisements, privilégient très largement, et donc beaucoup plus qu'autrefois, les espaces urbains au sens large (incluant le périurbain). Les zones d'activités regroupant des usines dans la proche périphérie urbaine, branchées sur les axes autoroutiers et bien reliées au reste du monde (fournisseurs et clients), sont une caractéristique territoriale de l'industrie d'aujourd'hui. La ville, en effet, concentre à la fois la main d'œuvre potentielle, les compétences (écoles, organismes de formation, universités), les consommateurs, les relais avec le reste du monde, réels (axes routiers, aéroports) et virtuels (connectivité), etc.

11 Ce lien n'est pas récent et, dès les temps proto-industriels, les villes comportent des quartiers où artisans et ouvriers à domicile se concentrent (vallée de la Bièvre à Paris Le Roux, 2010 ; les canuts lyonnais à la Croix-Rousse, etc.). Puis, avec les révolutions industrielles, les villes attirent des usines pour les raisons évoquées ci-dessus, déjà en œuvre alors pour la plupart. Cette configuration est particulièrement présente dans des branches industrielles légères et aval (confection, petite métallurgie, tréfilerie, constructions mécaniques, etc.) alors que d'autres branches, plus lourdes et dépendant plus de la matière première et de l'énergie (sidérurgie, métallurgie, chimie, filature, tissage, etc.) à une époque où les transports sont peu performants, s'installent au plus près des gisements. Dans ce cas, la main d'œuvre doit être amenée sur place et une forme particulière d'urbanisation peut ainsi se développer.

Dès lors que l'importance, à la fois historique et actuelle, des liens entre industrie et ville est posée, comment, dans une perspective de connaissance de l'héritage, de l'identité locale et d'aménagement (ou plutôt de réaménagement), reconnaitre, définir, caractériser, classer ${ }^{6}$, etc. les villes créées ou seulement transformées par l'irruption, le développement, puis les mutations, voire le déclin et la disparition, de l'industrie ? Ce 
questionnement est l'objet essentiel de ce numéro spécial de la RGE, autour de quatre pistes principales.

\section{A - Une question de genèse}

13 Les formes territoriales industrialo-urbaines sont donc d'abord liées à une question de genèse faisant une large part à l'histoire de l'organisme industrialo-urbain. En effet, le paragraphe introductif précédent a posé les deux principaux rapports entre la ville et l'industrie, détaillés ci-dessous :

\section{Ville industrielle}

Au delà de la présence proto-industrielle, la ville déjà constituée a accueilli l'industrie. Celle-ci s'installe en bordure de la ville d'alors, constituant des quartiers spécifiques, caractérisés par une grande hétérogénéité car ils mêlent usines, entrepôts, habitat intercalaire, petits commerces et bars, services divers, etc., le tout entrecoupé de nombreuses bretelles ferroviaires, bassins portuaires, etc. Ces quartiers en position identique des anciens faubourgs médiévaux (au contact du noyau urbain originel mais juste en dehors) forment donc des faubourgs industriels au paysage spécifique, certes marqués par les usines, mais aussi par un mélange industrie / habitat / services / commerces très peu planifié et chaotique, contrastant avec les quartiers urbains anciens.

Généralement utilisée pour faire référence, de façon générale, aux villes durant le pas de temps où l'industrie a été une activité économique dominante (XIX ${ }^{\text {ème }}$ siècle et première moitié du XX⿳亠丷⿵冂丶 ainsi faire référence à ces villes préexistantes à l'ère industrielle, mais qui ont accueilli, dans leurs faubourgs, l'activité industrielle. Elle a l'avantage d'être suffisamment large pour embrasser une grande variété de cas, mais toujours avec la présence d'une urbanisation préindustrielle. Cette marque de l'industrie est prolongée, après le tournant des Trente Glorieuses et de la crise du fordisme, par l'exurbanisation des usines et leur regroupement en parcs industriels prévus à cet effet (les Zones Industrielles - ZI - en France). Si ces regroupements planifiés de hangars fonctionnalistes, en périphérie de la ville d'alors, bien reliée aux échangeurs autoroutiers, diversifient très vites leurs activités (vers la logistique, les services aux entreprises, devenant donc plus des parcs d'activités - les Zones d'Activités , ZA, en France - que des parcs industriels), ils n'en restent pas moins des éléments paysagers très repérables autour de toutes les villes du monde, même si moins spécifiquement industriels que les faubourgs industriels les ayant précédés. La ville industrielle existe donc toujours, même si l'activité en question s'est, en se concentrant dans les parcs planifiés à cet effet, paradoxalement diluée dans un paysage d'entrée de ville dominée par les hangars fonctionnalistes banalisés à tout type d'activité et les lotissements pavillonnaires.

16 Au fil de cette histoire industrielle, notamment de la fin du XVIII ${ }^{\text {ème }}$ au début du XX ${ }^{\text {ème }}$ siècle, certaines de ces villes particulièrement attractives pour l'industrie, sont très transformées par cette dernière, même dans leur noyau central originel. L'industrie devient un nouveau squelette de la ville et le tissu urbain ancien intègre donc ateliers, usines, habitat ouvrier spécifique (c'est-à-dire des cités ouvrières ou des courées comme à Roubaix), infrastructures de transport, tout autant que de nouveaux quartiers 
encore plus spécifiquement marqués par l'industrie. Des villes comme Manchester, Roubaix, Turin ou encore Dunkerque, étudiée par Ch. Beaurain et Ch. Chembessi dans ce numéro sont dans cette configuration, à la limite entre la ville industrielle et le modèle suivant, la ville-usine.

\section{Ville-usine}

Le second modèle, celui de la ville-usine, se caractérise par un ensemble urbain né, à la différence du précédent où la ville préexistait à l'industrie, entièrement ou presque de la mine $e^{7}$ ou de l'usine lors de deux premières révolutions industrielles. L'expression apparaît chez l'historien et urbaniste P. Lavedan en 1941 dans son ouvrage « Histoire de l'urbanisme. Renaissance et Temps Modernes ", réédité en 1959. Elle réapparait plus tard dans d'autres de ses ouvrages comme «Histoire de l'urbanisme. Époque contemporaine» (p. 184 et p. 224 à propos d'Arvida) en 1952 ou, 30 ans plus tard en 1982, dans le collectif sous sa direction "L'urbanisme à l'époque moderne, XVI ${ }^{\text {ème }}$ XVIII ${ }^{\text {ème }}$ siècle ». Son utilisation est alors parallèle et indifférenciée à celle de ville industrielle. En effet, les auteurs indiquent en début de chapitre (p. 101) qu'ils étudieront, en seconde partie, la ville-usine, puis, arrivés à cette partie (p.111), l'intitulent "ville industrielle », pour, dans la même page un peu plus loin, reprendre l'expression ville-usine à propos des Salines Royales de Cl.-N. Ledoux à Arc-et-Senans dans le Doubs. Ils utilisent d'ailleurs indifféremment ville d'usine, cité industrielle, ville industrielle, ville-usine à de nombreuses reprises sans définir ces termes. Ville-usine même si ville d'usine ${ }^{8}$ serait peut-être encore mieux approprié - correspond pourtant bien à l'idée et à la représentation d'une ville entièrement née de l'industrie, autour d'une ou plusieurs usines qui ont fait naitre la ville. Reprise ensuite, dans plusieurs publications en géographie (Doyen, 1983 ; Bruyelle \& Dézert, 1983 ; Jalabert \& Grégoris, 1987), l'expression est plus récemment revisitée au prisme d'une approche par les paysages et la patrimonialisation (Edelblutte, 2010 ; Del Biondo \& Edelblutte, 2016).

La discussion est ouverte autour de l'utilisation du terme pour désigner ces organismes urbains nés plus ou moins totalement, plus ou moins rapidement, de façon plus ou moins aboutie... de l'industrie. De plus, comme le montrent M. Picon et G. Dorel-Ferré dans ce numéro, l'expression est déclinée en de nombreuses variétés, dont deux principales, là aussi liées à la genèse de ces organismes :

\section{Ville-usine non planifiée}

19 Les industriels s'installent près d'un gisement ou d'une source d'énergie, le plus souvent en milieu rural, à proximité d'un village agricole parfois marqué d'un vieux fond proto-industriel. La mine et/ou l'usine, voire les usines, grandissent, doivent faire venir des ouvriers et donc constituer un lieu d'habitation. Plus ou moins rapidement, avec une planification faible voire inexistante, car entravée par la présence du vieux village aux autorités déjà constituées et de plusieurs entreprises industrielles concurrentes, au moins pour la main d'œuvre et le foncier, l'ensemble peut atteindre la taille d'une ville à l'allure de nébuleuse informelle. Ces villes-usines non-planifiées sont les moins étudiées, les moins connues car moins facilement identifiables que les suivantes. 


\section{Ville-usine planifiée}

20 Sibérie, etc.), dans des espaces moins densément peuplés, européens comme américains (ou encore pour d'autres raisons, cf. infra), la ville est entièrement créée ex nihilo. Il s'agit donc ici d'une ville-usine totalement planifiée par un industriel aux côtés d'un gisement et/ou d'une source d'énergie. Dans ce cas, la ville est donc entièrement créée par une entreprise et l'expression ville d'entreprise peut aussi être utilisée. Ces company towns (Garner - dir., 1992), villes de compagnie ou villes d'entreprise, nombreuses en Amérique du Nord (L.K. Morisset, 2017), mais présentes partout dans le monde, sont des modèles urbains fascinants par leur jusqu'au-boutisme planificateur qui s'exerce tant sur le plan territorial que social et symbolique. M. Picon traite dans ce numéro des liens et ponts entre les modèles européens et nord-américains de ces villes-usines planifiées que sont les company towns. Là aussi, comme pour certaines villes industrielles qui évoluent vers le modèle de la ville-usine non-planifiée (cf. supra), les modèles ne sont pas étanches. La planification peut succéder à la non-planification (cas de villes minières roumaines, étudiées ici par G. Paşcu, lentement développées par le secteur privé, puis planifiées par les autorités communistes) ou, plus fréquemment, la planification initiale est brouillée par la multiplication du nombre d'acteurs planificateurs (les entreprises, les collectivités locales, l'État, etc.), comme le montre A. Brichler à propos de Bataville.

Dans ces villes-usines, planifiées ou non et bien au-delà d'une seule aventure économique et sociale, c'est d'une aventure culturelle qu'il s'agit. Les représentations, les symboles et les mythes sont bien présents, autour de la culture ouvrière, des luttes syndicales, de la figure paternaliste patronale, etc. La présence d'un leader patronal charismatique est d'ailleurs une condition essentielle pour le développement et la pérennité d'une ville née de l'industrie, car elle permet, notamment lorsque ce patron est aussi maire de la ville (Edelblutte, 2011), une identification de la nouvelle ville à l'entreprise et, par-delà, la naissance et le renforcement d'une identité locale d'autant plus forte chez les habitants-ouvriers. Dans ces villes-usines, comme dans les villes minières évoquées par S. Daviet en 2006, «le pouvoir industriel s'est longtemps substitué au pouvoir communal ou urbain traditionnel. La mine était maîtresse du sol, organisatrice des logements et des équipements, de la vie associative et culturelle, de la vie politique et syndicale, à travers l'appartenance des notabilités, comme d'une large part de la population, à la même communauté professionnelle» (p. 250). Cette fusion entre l'entreprise et la commune, entre l'économie et le politique, crée une identité propre, rendant la ville en question différente des autres villes (Jonas, 1985). Comme le montre L. Gauduchon à propos d'Épinac, ces villes construisent ainsi une identité à part, fort différente de celles des villes industrielles; identité particulière dont il faut tenir compte notamment au moment de la reconversion puis du redéveloppement territorial, questions abordées largement dans ce numéro par la plupart des auteurs, au prisme de l'enjeu environnemental (Ch. Beaurain \& Ch. Chembessi à Dunkerque), des jeux d'acteurs (É. Marochini dans la vallée de Fensch) ou des enjeux de mise en valeur patrimoniale (G. Dorel-Ferré). Cette question patrimoniale est parfois abordée dans des conditions plutôt favorables, liées à la qualité intrinsèque de la ville-usine et à l'histoire industrielle locale (Saltaire étudiée par A. Caignet) ou, au contraire, dans des conditions difficiles, comme par exemple la transition du communisme au capitalisme en 
Roumanie (G. Paşcu) ou encore l'isolement dans un espace du rural profond, comme Bataville (A. Brichler).

\section{B - Une question de taille et d'échelle}

23 Si la question de la genèse (et donc de l'histoire) de l'organisme industrialo-urbain est essentielle pour comprendre sa spécificité et donc pour réfléchir et agir quant à son redéveloppement après la crise, la question de la taille de l'ensemble provoque des débats qu'il n'est pas possible de trancher et donc de clore. Certes, l'approche historique permet de distinguer, comme le montre G. Dorel-Ferré, deux grandes périodes (l'une, la plus ancienne, dominée par des formes plus réduites: les villages ouvriers telles que les colonies industrielles catalanes; l'autre, plus récente, où ce sont les villes-usines qui dominent), mais la question de la limite entre la ville-usine, le village ouvrier, voire l'isolat industriel (Schwarz, 2019) ou l'usine-pensionnat (Nacé \& Nacé, 2008), stades inférieurs en taille au village ouvrier, reste posée et très difficile à définir.

Cette question de la distinction (et du passage) du village ouvrier à la ville-usine, ne peut pas être simplement liée au nombre d'habitants, d'autant plus que celui-ci, notamment en France, est tributaire des limites communales utilisées pour le recensement et donc de la définition de commune urbaine par l'Institut National de la Statistique et des Études Économiques (INSEE), à partir de 2000 habitants agglomérés sur un territoire communal. Ce seuil statistique par commune, déjà discutable en luimême notamment lorsque l'organisme industrialo-urbain s'étend sur plusieurs communes (cf. le cas de Bataville étudié par A. Brichler et détaillé à ce propos plus bas), ne permet pas d'intégrer dans la définition de la ville les nécessaires éléments de polarisation inhérents à l'attractivité urbaine : polarisations économique, sociale, commerciale, culturelle, politique... Comment mesurer, quantifier, tout cela à l'échelle des organismes industrialo-urbains ? Dans bien des cas, comme celui d'organismes assez fermés et seulement équipés de services de base telles les colonies industrielles catalanes étudiées par G. Dorel-Ferré en 1992, le caractère urbain est faible et le terme de village ouvrier s'impose. À l'autre bout du spectre, quelques exemples (Hayange et Uckange montrés par É. Marochini dans la vallée de la Fensch ou Jœuf, Arvida montrés par M. Picon) regroupent un ensemble de services et de commerces complets et variés et une population largement supérieure au seuil de 2000 habitants de l'INSEE et peuvent donc clairement être qualifiés de villes-usines. Cependant, les petits organismes miniers et industriels de la montagne roumaine, évoqués par G.Paşcu, Bataville analysé par A. Brichler ou encore Épinac étudié par L. Gauduchon, sont justement dans un entre-deux, plus tout à fait des villages ouvriers, pas encore complètement des villes-usines. Ils sont typiques de ces organismes dont l'évolution a $\mathrm{pu}$, à un moment, tendre vers une urbanisation claire, mais qui a été ralentie voire interrompue par le déclin, la crise et l'effondrement industrialo-économique.

À cette question de la taille s'ajoute aussi la question, particulièrement centrale pour les géographes, des échelles d'observation et de leurs relations. En effet, si certains ensembles très bien individualisés au cœur d'espaces ruraux ou pionniers (Bataville étudiée par A. Brichler, Arvida évoqué par M. Picon), certains organismes, pourtant bien reconnaissables en tant que villages ouvriers ou villes-usines à leur échelle, sont inclus dans des tissus urbains plus ou moins continus, complets et organisés. Analysée à 
une échelle locale, la forme urbaine caractéristique apparaît bien, mais si l'échelle d'observation est élargie, la ville-usine très planifié intègre une ville plus large constituée parfois de plusieurs de ces systèmes industrialo-urbains. C'est le cas de Saltaire, étudiée par A. Caignet. Inscrite par l'UNESCO au titre du patrimoine mondial et protégée par plusieurs dispositions nationales, en raison de son exceptionnelle cohérence, elle est en fait intégrée dans la ville de Shipley, elle-même partie du district métropolitain de Bradford, dans un ensemble au bâti continu et particulièrement hétéroclite. Il s'agit donc à l'échelle locale d'une ancienne ville-usine très planifiée, mais intégrée, à une échelle plus large, dans une conurbation textile développée dès le XVIII ${ }^{m e}$ siècle et juxtaposant, dans le plus grand désordre urbain et donc non-planifiée, de nombreux petits systèmes industrialo-paternalistes. La création de Saltaire est d'ailleurs liée aux difficultés nées de cette non-planification urbaine, puisque son créateur Titus Salt y a regroupé, en les exurbanisant à Shipley dans une préoccupation à la fois pragmatique et hygiéniste, plusieurs de ses anciennes petites usines textiles et logements ouvriers, auparavant dispersés dans Bradford. De même, les villes-usines de la vallée de la Fensch, étudiées par É. Marochini et initialement organisées autour d'usines séparées, intègrent, durant les Trente Glorieuses, un système industriel refondu, réorganisé et beaucoup plus vaste, s'étendant alors sur plusieurs communes. Ainsi nait la SOciété Lorraine de LAminage Continu (SOLLAC), une usine intégrée à l'échelle de toute la section aval de la vallée de la Fensch et s'étendant de Hayange à l'amont à Florange à l'aval, élargissant le système industrialo-urbain et donc la villeusine à l'échelon intercommunal. La Communauté d'Agglomération du Val de Fensch (CAVF), acteur essentiel de ce territoire, correspond d'ailleurs en grande partie à cette nouvelle organisation économique issue des Trente Glorieuses, avant le déclin et la restructuration en cours de l'usine, aujourd'hui propriété d'ArcelorMittal, dont seule la partie aval (Florange) fonctionne encore. Ces configurations, où des villages-ouvriers et villes-usines très planifiés à leur échelle sont intégrés par coalescence à des organismes urbains plus anciens ou créés par d'autres industriels, sont assez fréquentes et brouillent les définitions et représentations.

Enfin, même les villes-usines et villages ouvriers les mieux individualisés et repérables, car non intégrés dans un tissu urbain proche et/ou plus isolés, ont des liens avec des villes plus importantes, sensibles lorsque l'on change l'échelle d'observation: Arvida, dès les origines, est liée aux municipalités voisines de Jonquière et Chicoutimi; Bataville recrute jusqu'à Sarrebourg; les colonies industrielles catalanes étudiées par G. Dorel-Ferré sont liées commercialement et financièrement à Barcelone, etc. Ainsi, les définitions et délimitations de tous ces organismes industrialo-urbains doivent aussi tenir compte de cette question d'échelle qui, comme la taille de l'ensemble, peut permettre de trancher entre l'un ou l'autre modèle même si le doute subsiste parfois tant certains oscillent à la marge entre deux modèles.

\section{C - Une question d'environnement politique local et national}

La reconnaissance et la typologie de ces ensembles industriels est aussi liée à une question de politique qui se décline aussi en fonction de l'échelle, des pouvoirs locaux au type de régime politique.

Dans un régime libéral et capitaliste, la planification est, normalement, faible au niveau de l'État, mais très forte au niveau de l'entreprise, qui s'entend, au moins jusqu'à la fin 
du fordisme, à l'échelle du système industrialo-paternaliste (donc de la ville-usine et de ses variations où l'industriel exerce un pouvoir très fort). Ce système, surtout s'il est isolé (en front pionnier ou milieu très rural), est celui de la ville d'entreprise, villeusine très planifiée, où le contrôle exercé par l'entreprise déborde largement du domaine économique sur les domaines politiques, sociaux, culturels, etc. et est alors quasiment total. Cela va parfois jusqu'à l'utilisation d'une monnaie propre à la villeusine, celle de l'entreprise, comme le montrent L.K. Morisset et L. Noppen en 1994 à propos de la Price Brothers and Company pour la ville d'entreprise de Kénogami (Québec, Canada), créée autour d'une papeterie au début du $\mathrm{XX}^{\text {ème }}$ siècle.

Ce pouvoir presqu'absolu de l'industriel est d'autant plus fort qu'il s'exerce aussi dans le domaine politique. En effet, les pouvoirs politiques locaux sont alors exercés par les industriels qui sont élus, en France par exemple, aux fonctions de maire, conseiller général, voire député (Edelblutte, 2011). Cette confusion entre politique et économique est à la fois due, dans des communes uniquement agricoles à l'arrivée de l'industrie, au manque de personnel politique local qualifié face à l'urbanisation rapide du territoire et à la volonté de contrôle des industriels paternalistes sur « leur " population; elle ne peut, dans un premier temps, que renforcer la cohérence urbanistique de la ville d'entreprise qui prend alors tout son sens.

Cependant, en Europe occidentale, où la densité de population est forte, les ensembles industrialo-urbains bien identifiés, planifiés et cohérents à leur création, comme Saltaire étudié dans ce numéro par A. Caignet, Thaon-les-Vosges (Edelblutte, 2014), Port Sunlight près de Liverpool, Bournville près de Birmingham, etc. sont très vite entourés d'autres tissus urbains et/ou brouillés par la coalescence avec d'autres systèmes industrialo-urbains voisins et par l'intervention croissante des pouvoirs publics. La ville-usine tend alors à être de moins en moins planifiée, de plus en plus hétérogène. L'industriel perd peu à peu la main sur l'aménagement urbain, notamment lors des périodes de crise et de déclin industriel, avec le renforcement du pouvoir des collectivités locales et de la politique sociale et d'aménagement du territoire des États.

Cette tendance lourde vers une non-planification diffuse et de moins en moins contrôlée dans les ville-usines déclinantes est contrebalancée, après la seconde guerre mondiale, notamment en Europe occidentale, par le développement d'actions d'aménagement planificatrices, mais à d'autre échelles. L'État aménageur introduit en effet dans les villes le zonage des activités, concentrant les nouvelles usines dans les parcs industriels périphériques prévus à cet effet. Cette planification nouvelle est aussi lisible à l'échelle nationale. Par exemple, de grosses usines sidérurgiques sont installées sur le littoral, à l'initiative de l'État, pour relayer la sidérurgie de l'intérieur et développer des territoires en difficulté. C'est le cas des usines de Tarente, dans les Pouilles en Italie, de Fos-sur-Mer et de Dunkerque en France, dans les années 1960. L'État, mais plus sûrement les collectivités locales, participent alors à la construction de logements et de services, transformant les communes d'accueil, comme celle de Grande-Synthe, en banlieue de Dunkerque, évoquée par Ch. Beaurain et Ch. Chembessi dans ce numéro. De même, autour du complexe sidérurgique et industriel de Fos-surMer, une ville nouvelle, portée par l'Établissement Public d'Aménagement des Rives de l'Étang de Berre (EPAREB) et un Syndicat Intercommunal à Vocations Multiples (SIVOM), agissant en plus ou moins bonne entente avec les entreprises, est mise en place pour gérer l'afflux des ouvriers sur place (Tarrius, 1987). Toujours en France, la construction de logements et d'équipements de loisirs et de services autour des 
centrales nucléaires par Électricité De France (EDF) (Meyer, 2014), ou encore la mise en place de la très planifiée ville-usine de Mourenx, née dans les années 1950 sur le gisement de gaz naturel de Lacq dans les Pyrénées-Atlantiques, participent à ce modèle planifié.

À l'autre extrémité du spectre politique, les régimes communistes, par essence planificateurs et où l'État se substitue aux entreprises, ont multiplié les créations industrialo-urbaines ex nihilo, tant pour des raisons pragmatiques (éloignement de toute ville déjà constituée en régions extrêmes: Nord de la Russie, Sibérie, zone de montagne, etc.) qu'idéologiques. Ainsi, en Pologne, la ville-usine de Nowa Huta, créée en 1951 autour d'une usine sidérurgique, l'est autant pour assurer l'approvisionnement national en acier que pour contrer la vieille capitale catholique et conservatrice du pays, Cracovie, à quelques kilomètres de là. Il s'agit ainsi, par la construction de cette ville-usine d'État ultra planifiée, de créer un Homme nouveau sur des bases ouvrières et égalitaires. Si ces créations existent dans tous les pays d'Europe centrale communiste (Nowa Huta déjà citée, Eisenhüttenstadt en Allemagne de l'Est), parfois avec moins de systématisme résidentiel (usine sidérurgique de Šaca à côté de Košice en Slovaquie ; usine sidérurgique de Kremikovtsi près de Sofia en Bulgarie), l'URSS s'en était fait une spécialité, avec la constitution de villes-usines géantes autour d'usines non moins énormes. La sidérurgie a généré Magnitogorsk (440 000 habitants à l'apogée), née dans les années 1930 à côté d'un très important gisement de fer ; ou encore Togliatti (720 000 habitants à l'apogée), née dans les années 1960 autour de l'usine AvtoVaz fabriquant les automobiles Lada.

\section{D - Une question de méthodes}

33 La reconnaissance des formes industrialo-urbaines est aussi une question de méthodes. En effet, au regard des méthodes et pratiques propres à chacune des disciplines qui s'intéressent à l'industrie, reconnaitre et définir ces formes liant l'industrie à la ville est une question tout à la fois de données, d'enquêtes et d'entretiens, d'observation et d'analyse du paysage, de reconnaissances de terrain, d'étude d'archives et d'études de sources bibliographiques, etc.

34 À cet égard, la nécessité du varier et croiser les méthodes et parfaitement illustrée par le cas de Bataville, étudié par A. Brichler. L'analyse paysagère et géohistorique qu'il y développe permet en effet d'identifier assez facilement une ville-usine planifiée, ville d'entreprise presque caricaturale, là où une étude strictement quantitative n'aurait pu le faire. En effet, malgré les récentes réformes ${ }^{9}$, le découpage communal français est caractérisé à la fois par l'émiettement et l'ancienneté. Les limites communales sont certes fixées à la Révolution, mais s'ancrent dans un passé bien plus lointain, celui des paroisses et auparavant des finages, donc bien antérieur à l'industrialisation. Les industriels n'ont ni pu, ni voulu tenir compte, au moment de leur implantation, de ce découpage déjà vu comme obsolète (Edelblutte, 2006). Ainsi, quand Bata implante sa ville-usine en 1932, l'important est le site, choisi pour son bon accès au canal et à la voie ferrée. Les limites communales ne sont pas du tout prises en compte et l'ensemble s'étend sur trois communes, dont deux, Moussey et Réchicourt-le-Château, sont concernées par la zone habitée et donc recensée. Ces deux territoires communaux sont nés à la Révolution, calquées sur des paroisses et finages développés autour de vieux villages ou bourgs agricoles, détachées de Bataville de deux kilomètres pour l'un et de 
trois pour l'autre. Ainsi, la population de Bataville est statistiquement séparée en deux parts inégales et regroupée avec la population agri-rurale de deux villages différents dans deux communes différentes qui échappent ainsi aux "radars" statistiques ${ }^{10}$ habituels permettant de définir la commune urbaine, et donc la ville-usine. Inversement, l'analyse paysagère ne peut à elle seule appréhender l'ampleur réelle du système Bata local (Bata recrutait dans un rayon de plusieurs dizaines de kilomètres et tous les employés ne résidaient donc pas à Bataville) et mondial (Bataville s'inscrit dans le cadre de l'entreprise transnationale tchécoslovaque Bata et de son réseau de batavilles ; elle subit donc, entre autres, les contrecoups des évolutions géopolitiques de l'Europe centrale). Ainsi, les méthodes quantitatives, les enquêtes et entretiens, l'analyse de source bibliographiques, iconographiques, les déplacements sur le terrain... sont ici nécessaires tout autant que l'étude des paysages.

\section{Conclusion}

L'objectif de cet article introductif n'est donc surtout pas de trancher entre les approches mais plus sûrement de présenter différentes façons de faire pour reconnaître, étudier, analyser les formes industrialo-urbaines. Ce travail est indispensable pour les intégrer dans les problématiques territoriales, non seulement dans une perspective d'aménagement, mais aussi dans une nécessité de connaissance, notamment dans l'éventualité d'une préservation de l'héritage de ces villes-usines, de plus en plus souhaitée par les populations.

Tout en assumant les interrogations, voire les contradictions entre les résultats des méthodes et les définitions, la classification proposée dans la figure 1 tient compte de ces diverses approches et surtout des questionnements qu'elles apportent. Pouvant être croisée avec divers modèles proposés dans les articles du numéro, la figure 1 (et cet article introductif lui-même) espère ne pas figer la réflexion ni imposer un modèle universel, mais être utile à la réflexion et surtout à la reconnaissance de ces formes d'urbanisme et d'urbanité particulières que sont les villes-usines et leurs corolaires. Là est l'utilité des modèles qui sont destinés à être questionnés, voire remis en cause périodiquement, et ne constituent jamais des vérités révélées et intangibles; d'où la présence, sur la figure, de zones floues et de flèches illustrant des tendances. 
Figure 1 : Essai de typologie et de modèles à propos des formes industrialo-urbaines

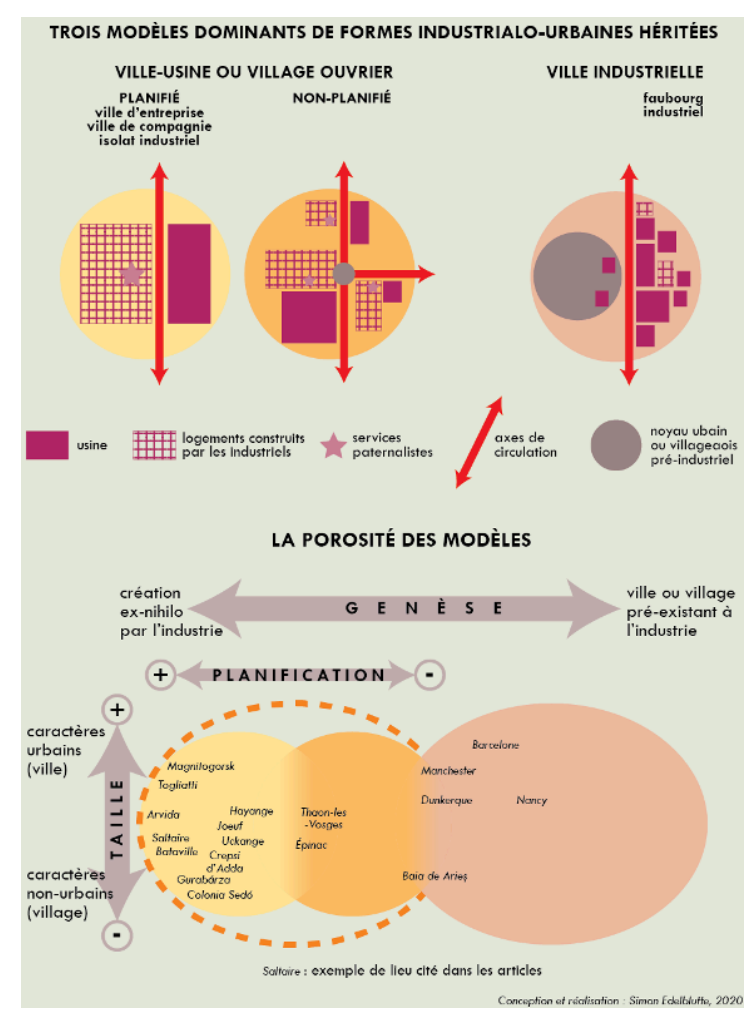

La nécessité et le besoin de classer et de modéliser peut d'ailleurs elle-même être aussi questionnée, notamment devant l'ampleur et la difficulté de la tâche et surtout le fait que ce classement laisse toujours une part d'ombre, une part de flou, une part de marge... dont la figure 1 tente néanmoins de tenir compte avec les zones d'intersection entre les types de formes industrialo-urbaines. Ainsi, la typologie peut rester opératoire (et donc permettre de réfléchir et d'analyser) justement à condition de souplesse et de discussion au regard des approches utilisées, notamment dans les «zones grises» (en fondu de couleurs sur la figure), les plus complexes mais aussi les plus passionnantes à l'étude.

Cette complexité est encore renforcée lorsque l'on tient compte des expressions utilisées en langue étrangère, dont les traductions renforcent encore la part de flou dans les termes désignant ces formes industrialo-urbaines. M. Picon ou encore G. DorelFerré dans leurs articles, évoquent ces difficultés liées aussi à la culture de différents pays. Un tableau de correspondance, d'une langue à l'autre, entre les termes et expressions, pourrait être, à l'échelle d'organismes internationaux tels que le Comité international pour la conservation du patrimoine industriel - The International Committee for the Conservation of Industrial Heritage (TICCIH), proposé. Enfin, l'industrie et la mine ne sont pas les seules à avoir généré des villes ou de vastes quartiers urbains planifiés : l'étude des villes militaires ou militarisées (Gaymard, 2014), des villes de religion (Chevrier, 2016), des villes ferroviaires (G. Dorel-Ferré évoque d'ailleurs dans son article le cas de Tergnier dans l'Aisne), des villes-campus anglo-saxonnes ou indiennes, etc. peut être mise en parallèle avec celle de villes-usines, les travaux se nourrissant les uns des autres.

Ainsi ce numéro de la RGE réunit-il, de l'étude plus générale à la plus particulière, huit articles qui sont autant de jalons apportés à la thématique de l'industrie et de la ville, 
loin d'être ainsi refermée. Il ne s'agit surtout pas, par ce numéro, de clore ici un débat, qui par sa diversité, sa transdisciplinarité et son internationalisation croissante, ne peut qu'être bénéfique à la recherche et à l'aménagement du territoire, au travers de la connaissance toujours plus grande de ces formes industrialo-urbaines particulières, fascinantes et passionnantes.

\section{BIBLIOGRAPHIE}

Benko G., 2008, La géographie économique : un siècle d'histoire, Annales de Géographie, $\mathrm{n}^{\circ}$ 664, p. 23-49.

Bergeron L. \& Dorel-Ferré G., 1996, Le patrimoine industriel, un nouveau territoire, Paris, Liris, 127 p. Blanc J.-N., 1991, Les friches industrielles de l'économique à l'urbain, Revue de Géographie de Lyon, vol. 66, $\mathrm{n}^{\circ} 1, \mathrm{p} .103-107$

Bost F., 2014, La France : mutations des systèmes productifs, Paris, Sedes, 253 p.

Boulay G. \& Grandclément A., 2019, Introduction à la géographie économique, Paris, Armand Colin, $222 \mathrm{p}$.

Bretagnolle A., Baudet-Michel S., Glita I. et Paulus F., 2019, Effondrement, résistances et transformations de l'emploi textile dans les villes françaises des années 1960 aux années 2010, Géographie, économie, société, 2019/4 (Vol. 21), p. 317-344. https://www.cairn.info/revuegeographie-economie-societe-2019-4-page-317.htm

Bruyelle P. \& Dézert B., 1983, Les relations entre la ville et l'industrie : formes anciennes et formes nouvelles, Hommes et Terres du Nord, $\mathrm{n}^{\circ}$ 1, p. 19-23.

Chardonnet J., 1965, Géographie industrielle. Tome II : l'industrie, Paris, Sirey, 461 p.

Chevrier M.-H., 2016, Pèlerinage, développement urbain et mondialisation : l'exemple de Lourdes ", Géoconfluences, mis en ligne le 19 octobre 2016. http://geoconfluences.ens-lyon.fr/ informations-scientifiques/dossiers-thematiques/fait-religieux-et-construction-de-l-espace/ corpus-documentaire/pelerinage-lourdes

Claval P., La Géographie culturelle, Paris, Nathan, 384 p.

Daviet S., 2005, Industrie, culture, territoire, Paris, L'Harmattan, 208 p

Daviet S., 2006, L'évolution du concept de reconversion : de la substitution des activités au redéveloppement des territoires. In : Daumalin X., Daviet S. \& Mioche Ph. - dir., Territoires européens du charbon, des origines aux reconversions, Aix-en-Provence, Publications de l'Université de Provence, p. 243-255.

Del Biondo L. \& Edelblutte S., 2016, Le paysage des anciennes villes-usines européennes : un nouveau patrimoine entre négation, alibi, reconnaissance et complexité des jeux d'acteurs, Annales de Géographie, ${ }^{\circ} 711$, p. 466-489.

Deshaies M., 2005, Exploitation minière et paysages, Habilitation à Diriger des Recherches, Université Nancy2, 481 p. 
Dézert B., 1992, Avant-propos. Les nouveaux dynamismes dans les régions anciennement industrialisées, Revue Belge de Géographie, fasc. 51, n 1-4, p. 3-4.

Donze J., 2001, L'Argentière-la-Bessée, de la reconversion au redéveloppement, Hommes et Terres du Nord, n 1, p. 39-45.

Dorel-Ferré G., 1992, Les colonies industrielles en Catalogne, le cas de la colonia Sedó d'Esparreguera, Paris, Éditions Arguments.

Doyen J.-P., 1983, Les villes-usines de la moyenne Moselle, Annales de la Société d'Émulation des Vosges, p. 52-71.

Edelblutte S., 2006, Géohistoire de l'adaptation de la maille communale à l'organisation spatiale. L'exemple des villes-usines. In : Bleton-Ruget A., Commerçon N., Gonod Ph. - dir., Territoires institutionnels, territoires fonctionnels, Institut de Recherche du Val de Saône-Mâconnais, p. 239-252.

Edelblutte S., 2006, Renouvellement urbain et quartiers industriels anciens : l'exemple du quartier Rives de Meurthe / Meurthe-Canal dans l'agglomération de Nancy, Revue Géographique de l'Est, tome XLVI, n³-4, p. 141-152. https://journals.openedition.org/rge/1455

Edelblutte S., 2010, Paysages et territoires de l'industrie en Europe : héritages et renouveaux, Paris, Ellipses, $272 \mathrm{p}$.

Edelblutte S., 2011, Paternalisme et territoires politiques dans la France de la seconde révolution industrielle. Un regard rétrospectif sur les liens entre firmes et territoires communaux, Revue Géographique de l'Est [En ligne], vol. 50 / 3-4 | 2010, mis en ligne le 17 octobre 2011. https:// journals.openedition.org/rge/3043

Edelblutte S., 2014, Reconversion industrielle ou redéveloppement territorial ? L'exemple de Thaon-les-Vosges, ancienne ville-usine textile lorraine, Géoconfluences, mis en ligne le 2 décembre 2014. http://geoconfluences.ens-lyon.fr/informations-scientifiques/dossiers-regionaux/lafrance-des-territoires-en-mutation/articles-scientifiques/reconversion-industrielle-ouredeveloppement-territorial-lexemple-de-thaon-les-vosges-ancienne-ville-usine-textile-lorraine

Fagnoni É., 2001, La mise en tourisme des friches industrielles en Lorraine : approche patrimoniale. In : Damien M.-M. \& Sobry C. - dir., Le tourisme industriel : le tourisme du savoir-faire?, Paris, L'Harmattan, p. 217-258.

Fagnoni É., 2005, Patrimoine et vieilles régions industrielles ; des territoires entre mémoire et projet. In : Gravari-Barbas M. - dir., Habiter le patrimoine. Enjeux, approches, vécu, Rennes, Presses Universitaires de Rennes, p. 565-579.

Fol S. \& Cunningham-Sabot E., 2010, « Déclin urbain » et Shrinking Cities : une évaluation critique des approches de la décroissance urbaine, Annales de Géographie, nº 674, p. 359-383.

Garner J. - dir., 1992, The Company Town: Architecture and Society in the Early Industrial Age, New York, Oxford University Press, 256 p.

Gasnier M. \& Lamard P. - dir., 2007, Le patrimoine industriel comme vecteur de reconquête économique, Panazol, Lavauzelle, 198 p.

Gaymard M.-C., 2015, Le patrimoine militaire sur la scène urbaine : les processus de reconversion des sites militaires à Metz, Revue Géographique de l'Est [En ligne], vol. 54 / n³-4 | 2014, mis en ligne le 11 février 2015. http://journals.openedition.org/rge/5330

George P., 1956, Précis de géographie économique, Paris, Presses Universitaires de France, 400 p. George P., 1978, Les méthodes de la géographie, Que-Sais-Je ?, Paris, Presses Universitaires de France, $126 \mathrm{p}$. 
Gravari-Barbas M. - dir., 2005, Habiter le patrimoine. Enjeux, approches, vécu, Rennes, Presses Universitaires de Rennes, p. 511-516.

Grésillon B., 2011, La culture comme alternative au déclin : mythe ou réalité ? Le cas des villes rétrécissantes allemandes, Géocarrefour, vol. 86, n² 2, p. 151-160.

Grossetti M, Beslay Ch., Daynac M., Guillaume R., Salles D. \& Tautelle F., 1998, La construction des politiques locales. Reconversions industrielles et systèmes locaux d'action publique, Paris, L'Harmattan, $224 \mathrm{p}$.

Grusti J., 1991, Cinq ans après. In : DATAR \& Ministère de l'Aménagement du Territoire et des Reconversions, La réhabilitation des friches industrielles, Paris, La Documentation Française, $45 \mathrm{p}$.

Guglielmo R., 2008, Pierre George, promoteur de la géographie industrielle, Cahiers de Géographie du Québec, n 146, p. 255-259.

Hau M., 2019, Pourquoi l'Allemagne a-t-elle moins souffert de la désindustrialisation ?, Revue du Rhin Supérieur, $\mathrm{n}^{\circ} 1$, p. 21-40.

Jalabert G. \& Grégoris M., 1987, Turin : de la ville-usine à la technopole, Annales de Géographie, $n^{\circ} 538$, p. 680-704.

Janin Cl. \& Andres L., 2008, Les friches : espaces en marge ou marges de manœuvre pour l'aménagement des territoires ?, Annales de Géographie, n 663, p. 62-81.

Jonas S., 1985, La ville industrielle : question d'identité, Revue Géographique de l'Est, t. XXV, n 2-3, p. 231-240.

Lamard P., Vitoux M.-Cl. - dir. \& Gasnier M. - coll., 2006, Les friches industrielles, point d'ancrage de la modernité, Panazol, Lavauzelle, $224 \mathrm{p}$.

Lavedan P., 1941, Histoire de l'urbanisme. Renaissance et Temps modernes, Paris, Laurens, 504 p. Lavedan P., 1952, Histoire de l'urbanisme. Époque contemporaine, Paris, Laurens, 446 p.

Lavedan P., 1959 (rééd.), Histoire de l'urbanisme. Renaissance et Temps modernes, Paris, Laurens, $530 \mathrm{p}$.

Lavedan P., Hugueney J. \& Henrat Ph., 1982, L'urbanisme à l'époque moderne, XVI ème - XVIII ${ }^{\text {ème }}$ siècles, Genève, Droz, $312 \mathrm{p}$.

Le Roux T., 2010, Une rivière industrielle avant l'industrialisation : la Bièvre et le fardeau de la prédestination, 1670-1830, Géocarrefour, vol. 85/3, 2010, p. 193-207.

Levet J.-L. - dir., 2012, Réindustrialisation, j'écris ton nom, Paris, Éditions Fondation Jean Jaurès, $349 \mathrm{p}$.

Manzagol Cl., 1980, Logique de l'espace industriel, Paris, Presses Universitaires de France, 248 p.

Mérenne-Schoumaker B., 2011, La localisation des industries. Enjeux et dynamiques, Rennes, Presses Universitaires de Rennes, 263 p.

Meyer T., 2014, Du « pays perdu » du Blayais à l' « émirat de Saint-Vulbas » : les territoires de dépendance au nucléaire en France, Hérodote, n¹55, p. 153-169.

Montagné-Villette S., 2001, Le secondaire est-il soluble dans le tertiaire ?, Annales de Géographie, $\mathrm{n}^{\circ} 617$, p. 22-37.

Morisset L.K. \& Noppen L., 1994, Jonquière, mémoires et lieux : Guide d'excursion et d'interprétation du patrimoine. Jonquière, Ministère de la culture et des communications, $103 \mathrm{p}$. 
Morisset L.K., 2017, Les « villes de compagnie » du Canada. Un patrimoine urbain pour le vivre ensemble de notre siècle ?, Entreprises et histoire, ${ }^{\circ}$ 87, p. 39-50.

Nacé Ch. \& Nacé J.-R., 2008, L'Ardèche, terre de patrimoine industriel ?, Historiens et Géographes, $n^{\circ} 401$, p. $277-284$.

Roth H., 2011, Les villes « rétrécissantes » en Allemagne, Géocarrefour, n² 2/75, p. 75-80.

Savall H., Zardet V., Bonnet M. - dir., 2013, Réindustrialisation et dynamisation multi-sectorielle, Paris, Economica, $244 \mathrm{p}$.

Schwarz F., 2019, Les isolats industriels en Alsace. In : Dorel-Ferré G. - Éd., Le patrimoine industriel dans tous ses états. Un hommage à Louis Bergeron, Université de Savoie-Mont Blanc, Chambéry, p. 256-265.

Stafford H. A., 2003, Industrial Geography in the United States, the past half century, The Industrial Geographer, vol. 1, Issue 1, p. 3-15. http://igeographer.lib.indstate.edu/stafford.pdf

Talandier M. \& Pecqueur B., 2018, Renouveler la géographie économique, Paris, Economica \& Anthropos, $296 \mathrm{p}$.

Tarrius A., 1987, Fos-sur-Mer. La diaspora des sidérurgistes lorrains, Les Annales de la Recherche Urbaine, 35-36, p. 31-40.

Touraine A., 1969, La société post-industrielle. Naissance d'une société, Paris, Denoël, 319 p.

Veschambre V., 2008, Traces et mémoires urbaines - Enjeux sociaux de la patrimonialisation et de la démolition, Rennes, Presses Universitaires de Rennes, 315 p.

Woronoff D., 1989, L'archéologie industrielle en France : un nouveau chantier, Histoire, économie et société, $8^{\mathrm{e}}$ année, $\mathrm{n}^{\circ} 3$ - L'industrialisation. p. 447-458.

\section{NOTES}

1. Comme par exemple le forum "L'usine et le territoire ", organisé par le journal Libération le 26 novembre 2019 à Toulouse, avec, entre autres, un débat intitulé « Réindustrialiser ? Quelle place pour l'industrie dans nos territoires ? ", auquel participait le géographe F. Bost.

2. Certains États, comme l'Allemagne par exemple, tant par choix politiques, caractéristiques mêmes de son tissu industriel (domination des biens d'équipements, montée en gamme précoce, etc.) que pour des raisons culturelles (liens historiques des Allemands avec la mine, puis l'industrie ; savoir professionnel valorisé, etc. - Hau, 2019), conservent un base industrielle forte.

3. "In the 1960's there was a concerted move to examine how companies organize space. These create the geography of the firm" (traduction libre de S. Edelblutte).

4. "There is still the gap between location theory and actual distributions" (traduction libre de S. Edelblutte).

5. La commission « industrie et emplois » du CNFG est renommé « géographie économique " en 2015, illustrant à la fois l'élargissement nécessaire de l'étude de la seule industrie au sein de l'économie entière, mais aussi une sorte de banalisation, sensible aussi dans ses formes, de l'industrie au sein de l'économie.

6. Le besoin et la nécessité de classer et de réaliser des typologies seront abordés et discutés en conclusion de cet article.

7. Les systèmes territoriaux construits par les mines et/ou les usines sont très proches. Ici, il a été choisi de ne pas différencier ville minière ou ville-mine (présentées par exemple dans l'article 
de G. Paşcu en Roumanie ou dans celui de L. Gauduchon avec l'exemple d'Épinac en France) de la ville-usine.

8. Sur une suggestion de L.K. Morisset et d'ailleurs déjà utilisé par P. Lavedan en 1952 (p. 183).

9. Après la loi de 1971 favorisant les regroupements communaux, dite loi Marcellin et considérée comme un échec, les lois Pélissard, (2015) Sido (2016) et Gatel (2019) semblent avoir enclenché une réelle dynamique de réduction du nombre de communes et donc une simplification du découpage communal français... mais le recul manque encore pour en être sûr.

10. Ces exemples d'usines installées sur une commune, voire plusieurs communes, avec les cités ouvrières tout ou partie sur une autre, sont nombreux. Ils ne condamnent pas une approche statistique très utile pour un premier balayage (Bretagnolle et al., 2019), mais montrent qu'elle doit toujours être confrontée au terrain et à d'autres méthodes.

\section{RÉSUMÉS}

Cet article introductif a pour objectif de présenter la thématique et les huit articles de ce numéro spécial de la " Revue Géographique de l'Est ». Il s'agit ici d'analyser les liens entre l'industrie et la ville, sur un temps long, dans une géohistoire des formes industrialo-urbaines, selon des approches et méthodes variées et sur des terrains multiples et à échelles différentes (Grand Est, France, Roumanie, Royaume-Uni, Espagne, États-Unis...). Les termes de ville-usine, village ouvrier, ville industrielle, etc. sont discutés et remis dans le contexte de l'évolution de la géographie économique et industrielle, avant que les différentes approches des huit auteures et auteurs soient présentées.

This paper aims to present the theme and the eight papers of this special issue of the "Revue Géographique de l'Est". It analyzes the links between industry and the city, over a long time, as a geohistory of industrial-urban forms. Methods and approaches used, as territories (Grand Est, France, Romania, United Kingdom, Spain, United States...) and scales of study, are varied. The terms of factory town, workers' village, industrial city, etc. are discussed and put back in the context of the evolution of economic and industrial geography, before the different approaches of the eight authors are presented.

\section{INDEX}

Mots-clés : Industrie, Ville-usine, Ville d'entreprise, Ville industrielle, Village ouvrier, Géographie industrielle, Géohistoire

Keywords : Industry, Factory town, Company town, Industrial city, Workers' village, Industrial Geography, Geohistory

\section{AUTEUR}

\section{SIMON EDELBLUTTE}

Université de Lorraine, LOTERR, Campus Lettres et Sciences Humaines, 23, boulevard Albert I $\mathrm{I}^{\mathrm{er}}$, 54000 NANCY - simon.edelblutte@univ-lorraine.fr 